\title{
Blood and cardiovascular health parameters after supplementing with ketone salts for six weeks
}

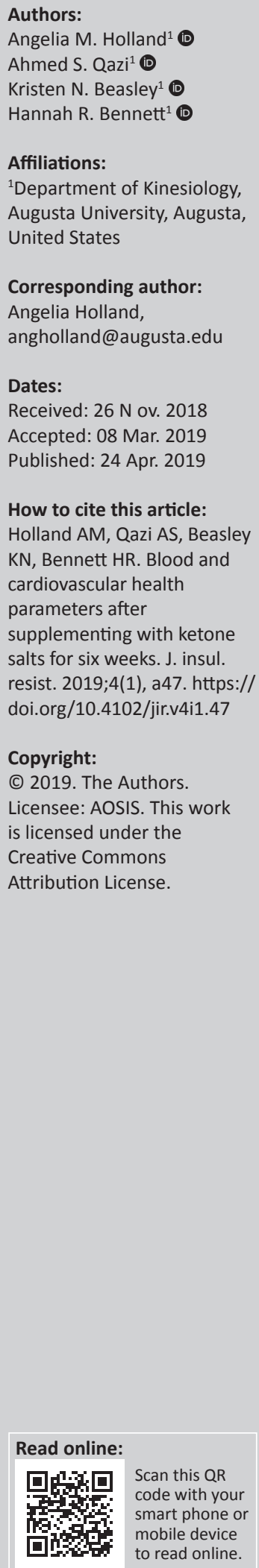

Background: Exogenous ketone salts (KS) have been administered as treatment for various health conditions; however, the safety of chronic supplementation in a healthy population has yet to be explored.

Aim: This study examined the safety of KS supplementation for 6 weeks in healthy, young adults and determined the effects of KS on blood ketone levels.

Setting: Data collection occurred in a laboratory at Augusta University.

Methods: Twenty-three men and women (aged 18-35 years old) supplemented with KS or a placebo (PLA) twice per day for 6 weeks in a randomised, double-blinded, PLA-controlled design. Baseline and post-intervention measures included body mass index (BMI), resting blood pressure and heart rate, questionnaires assessing mood and energy, urinalysis, and venous blood measures, including comprehensive metabolic panel (CMP), lipid panel, and complete blood count (CBC). In addition, the participants consumed the assigned supplement during the baseline and post-intervention visits; blood ketone levels were assessed immediately before and after 30 and $60 \mathrm{~min}$ post-supplementation.

Results: Systolic blood pressure was significantly lower $(p<0.05)$ after supplementing with KS for 6 weeks but not PLA. All other health parameters remained unchanged by KS supplementation, including BMI, resting heart rate, urinalysis, CMP, lipid panel, and CBC. After acute administration of KS but not PLA, blood ketone levels were significantly elevated $(p<0.001) 30$ and $60 \mathrm{~min}$ after supplementation at both baseline and post-intervention visits.

Conclusion: Chronic KS supplementation seems safe, significantly elevates blood ketone levels 30 and 60 min after supplementation and may lower blood pressure. Future explorations should determine the success of KS supplementation as a strategy to combat hypertension.

Keywords: blood pressure; complete blood count; comprehensive metabolic panel; cholesterol; beta-hydroxybutyrate; ketosis.

\section{Introduction}

A very low-carbohydrate, ketogenic diet (KD) has proven to be a successful holistic therapy for a range of metabolic and neurological disorders. ${ }^{1,2}$ Cellular utilisation of ketone bodies is often noted as the mechanism responsible for the therapeutic nature of a KD.,4 When glucose levels are too low to sufficiently supply bioenergetic requirements, the liver produces ketone bodies as an alternate fuel source. ${ }^{5}$ Ketone bodies are particularly important for tissues unable to utilise fat for energy, such as the brain. ${ }^{6,7}$ The physiological state of ketosis occurs in response to chronically elevated circulating ketone levels; ketosis is associated with fat loss, improved serum health markers (i.e. greater high-density lipoprotein cholesterol [HDLc] and lower triglycerides) and enhanced mitochondrial function. $8,9,10$

Ketone bodies serve as an efficient fuel source for most extra-hepatic tissues and include $\beta$-hydroxybutyrate $(\beta-\mathrm{HB})$, acetoacetate and acetone. ${ }^{6,11}$ Although the elevation in ketone bodies resulting from a KD has repeatedly demonstrated success in improving specific health conditions in both humans (i.e. epilepsy and polycystic ovary syndrome) and rodents (i.e. traumatic brain injury), adherence to a KD may be difficult for individuals to maintain because of its restrictive nature. ${ }^{12,13,14}$ Therefore, $\beta$-HB has become available in various exogenous supplemental forms as a non-restrictive nutrient strategy to elevate circulating $\beta$-HB levels and possibly reproduce the health benefits associated with a KD. ${ }^{15}$ Recent studies demonstrate similarities between a KD and exogenous ketone supplementation in both therapeutic and athletic performance outcomes. . $^{14,16,17,18}$ 
However, research on the safety of exogenous ketone salt (KS) supplementation is limited, especially in the adult human population. ${ }^{14,19}$ A few infant clinical case studies have shown $\mathrm{KS}$ administration to be safe and therapeutically effective for genetic metabolic conditions. ${ }^{20,21,22}$

Although no adverse events were reported in the infant case studies, it is important to determine the safety status of KS before examining their efficacy in treating health conditions. Therefore, the primary purpose of this study was to determine if chronic consumption of exogenous KS is physiologically safe for healthy, young adults. In addition, blood ketone concentrations were assessed for $1 \mathrm{~h}$ after KS supplementation to determine the supplement's effects on blood ketone levels. Based on previous case studies, we hypothesised that increasing blood ketone levels chronically via a 6-week exogenous KS supplementing regimen would not negatively alter blood or urine health markers.

\section{Methods \\ Experimental design}

A randomised, double-blinded, placebo (PLA)-controlled design was used to examine the safety of chronic KS consumption in healthy, college-aged adults. This study was conducted at Augusta University (AU; Augusta, GA). The supplements were sent to the primary investigator (PI) in white packages labelled ' $\mathrm{A}$ ' or ' $\mathrm{B}$ '; the PI, subinvestigators and participants were blinded until poststatistical analysis at the completion of the study. Prior to setting dates to consent participants, participant numbers 1-26 were manually randomised to supplement ' $\mathrm{A}$ ' or ' $\mathrm{B}$ ' by the PI and sub-investigators. Thirteen ' $A$ ' and 13 ' $B$ ' labels were mixed together, and an investigator blindly picked a label to pair with each subject number in order from 1 to 26. After randomisation, participants were assigned a participant number in the order in which they were familiarised by the PI.

Participants visited the laboratory for familiarisation and then a total of seven times. The informed consent document was signed during the familiarisation visit, baseline measures were taken during the first visit, weekly supplements were provided during the second-sixth visits and post-intervention measures were taken during the seventh visit. The study was approved by AU's Institutional Review Board, and all procedures performed were in compliance with the institutional guidelines.

\section{Participants}

Twenty-six overtly healthy male and female adults, aged 18-35 years old, were recruited between July and October 2017 from AU and the surrounding areas for this study. Exclusion criteria included (1) chronic smoking; (2) taking medications that affect cholesterol, blood pressure, insulin or renal function; (3) metabolic syndrome factors or (4) current pregnancy. Three participants dropped out after signing the informed consent (Figure 1).

Baseline characteristics for each supplemental group were reported for the 23 participants ( 11 women and 12 men) who completed the study (Table 1).

\section{Protocol}

During the familiarisation visit, the participants signed the informed consent document, filled out a health history questionnaire, took a pregnancy test if female and were advised to fast for $10 \mathrm{~h}$ (only water was allowed), and withhold from exercise, caffeine and nicotine for $12 \mathrm{~h}$ prior to the first visit. The first visit consisted of baseline measures, including a venous blood draw, urine sample, resting blood pressure and heart rate, questionnaires, and a finger prick for blood ketone levels. Participants then consumed their assigned supplement and blood ketone levels were measured 30 and $60 \mathrm{~min}$ after finishing the supplement. Participants received 7 days' worth of their assigned supplement as well as a supplement log. During the second-sixth visits, supplements and supplement logs were provided weekly. The seventh visit included postintervention measures and followed the same protocol as the first, baseline visit.

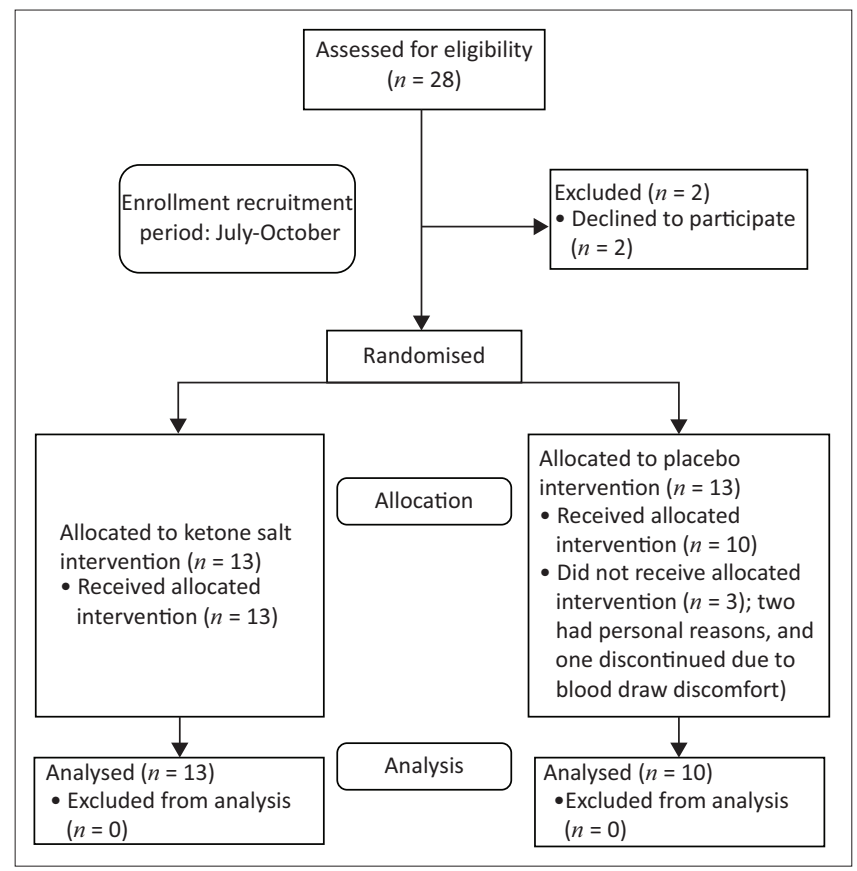

FIGURE 1: Flow diagram demonstrating number of participants recruited, enrolled and analysed.

TABLE 1: Baseline values for participants in the ketone salt and placebo groups.

\begin{tabular}{lcc}
\hline Characteristic & KS $(\boldsymbol{n}=13)$ & PLA $(\boldsymbol{n}=10)$ \\
\hline Age (years) & $24.8(4.8)$ & $23.3(3.3)$ \\
Sex & 7 males, 6 females & 5 males, 5 females \\
Height $(\mathrm{cm})$ & $172.8(7.7)$ & $170.7(7.7)$ \\
Weight $(\mathrm{kg})$ & $78.9(11.0)$ & $76.1(15.7)$ \\
\hline
\end{tabular}

Note: Values represent the mean ( \pm standard deviation).

*, represents significant difference between KS and PLA for age, height, and weight at $p<0.05$. KS, ketone salt; PLA, placebo. 


\section{Supplement}

Participants were randomised to either consume a KS supplement or the PLA in conjunction with a relatively normal diet (i.e. no dietary restrictions were implemented). The KS and PLA were flavour- and calorie-matched, and both in powder form. The participants were instructed to take two packets a day mixed with water, once in the morning and once in the afternoon, for 6 weeks. The KS included $7 \mathrm{~g}$ of beta-hydroxybutyrate combined with erythritol, L-Taurine, fermented L-Leucine, citric acid, natural flavour, vegetable juice colour, stevia, xanthan gum, beta carotene and approximately $920 \mathrm{mg}$ of sodium (KETO/ /OS® MAX, Prüvit Ventures, Inc., Melissa, TX). The PLA included maltodextrin and similar colour, sodium and flavouring as the KS. Both supplements contained $41 \mathrm{kcal}$ per serving. The participants filled out a weekly supplement log to confirm two servings of the assigned supplement were taken daily and to disclose other supplements consumed.

\section{Venous blood measures}

Venous blood samples were collected by a certified nurse at AU's Clinical Trials Office. Blood was drawn into one ethylenediaminetetraacetic acid (EDTA) anticoagulant sealed vacutainer, and two lithium heparin sealed vacutainers and then immediately transported to the Clinical Pathology Laboratory at AU's Hospital for subsequent analyses. Analyses included a comprehensive metabolic panel (CMP), lipid panel and complete blood count (CBC) for each participant. Comprehensive metabolic panel included plasma sodium, potassium, chloride, $\mathrm{CO}_{2}$, blood urea nitrogen (BUN), creatinine, calcium, glucose, total protein, albumin, total bilirubin, alkaline phosphatase, aspartate aminotransferase (AST) and alanine transaminase (ALT) levels. Lipid panel included plasma levels of total cholesterol (TC), HDLc, triglycerides (TG) and calculated low-density lipoprotein cholesterol (LDLc), if TG levels were $\leq 400 \mathrm{mg} / \mathrm{dL}$. The CMP and lipid panel were performed on an automated chemistry analyser (ADVIA 1800 Chemistry System; Siemens Healthineers, Erlangen, Germany). The CBC was analysed with a cellular analysis system (UniCel DxH 800 Hematology Analyzer Workstation; Beckman Coulter, Brea, CA) which included whole blood levels of white blood cells (WBC), red blood cells (RBC), haemoglobin $(\mathrm{Hgb})$, haematocrit $(\mathrm{Hct})$, mean corpuscular haemoglobin $(\mathrm{MCH})$, mean corpuscular haemoglobin concentration (MCHC), mean corpuscular volume (MCV), red cell distribution width percent (RDW), platelets (PLT) and mean platelet volume (MPV).

\section{Urine measures}

Urine samples were collected in sterile urine containers and transported immediately to the Clinical Pathology Laboratory for a dipstick chemical analysis (CLINITEK Novus Automated Urine Chemistry Analyzer; Siemens Healthineers, Erlangen, Germany). The urinalysis included blood, glucose, urobilinogen, ketones, leukocytes, $\mathrm{pH}$ and protein levels.

\section{Body composition and cardiovascular measures}

Height was measured during the first visit with a wall stadiometer (Stadi-o-meter; Novel Products, Inc., Rockton, IL, USA). Weight was measured with a beam balance scale (Healthometer; Continental Scale Corporation, Bridgeview, IL), and resting blood pressure and heart rate were measured with an automated blood pressure cuff (10 Series Upper Arm Blood Pressure Monitor; Omron Healthcare, Inc, Kyoto, Japan) during the first and seventh visits.

\section{Blood ketone levels}

$\beta$-Hydroxybutyrate levels were measured via a finger prick using a ketone meter (Precision Glucose Ketone Meter; Abbott Laboratories, Abbott Park, IL) at three time points during the visits 1 and 7. The first time point occurred immediately prior to consuming the assigned supplement (KS or PLA mixed with $12 \mathrm{oz}$ of water) and the second and third time points occurred 30 and $60 \mathrm{~min}$ after consuming the supplement, respectively.

\section{Questionnaires}

The Profile of Moods (POMS) and Visual Analogue Scale (VAS) questionnaires were filled out during the first and seventh visits. The POMS included a list of 58 descriptors of how one may feel (i.e. tense, sad, sluggish, cheerful, active and so on) with a rating scale of $0-4(0=$ not at all, 4 = extremely). ${ }^{23}$ The VAS included a numeric intensity scale of $1-10$ ( 1 = lowest, $5=$ neutral, $10=$ highest $)$ for the following variables: Hunger, satiety, mood, energy, focus and alertness.

\section{Statistical analysis}

The sample number of $10-13$ per group was predetermined based on participant numbers in similar studies. All statistical analyses were performed at the completion of the study, with no interim analyses, using SPSS (Version 25.0; IBM Corp., Armonk, NY). Baseline characteristics were analysed using two-way $t$ tests. All other variables, except $\beta$-HB levels, were analysed using a $2 \times 2$ (condition $\times$ time) repeated measures ANOVA. Conditions included KS and PLA and times included baseline and post-intervention. $\beta$-Hydroxybutyrate levels were analysed using a $2 \times 6$ (condition $\times$ time) repeated measures ANOVA in which conditions included KS and PLA and times included the three time points (baseline, 30- and 60-min post-supplement consumption) for visits 1 and 7 . Significance was set at $p<0.05$. If there was a significant condition $\times$ time interaction, a Bonferroni post hoc test was used to compare baseline and post-intervention outcomes within each condition.

\section{Ethical considerations}

The study was approved by AU's Institutional Review Board, and all procedures performed were in compliance with the institutional guidelines. 


\section{Results \\ Body mass index}

There was no main effect of time $(F=0.18, p=0.674)$ and no condition $\times$ time interaction $(F=0.45, p=0.510$; baseline: [KS] $27.6 \pm 3.4$ vs. [PLA] $26.3 \pm 2.9$ and post-intervention: $[\mathrm{KS}] 27.5$ \pm 3.7 vs. [PLA] $26.5 \pm 2.8$ ) for body mass index.

\section{Cardiovascular measures}

There was a no main effect of time for resting systolic blood pressure $(F=3.43, p=0.079)$, but there was a condition $\times$ time interaction $(F=4.67, p=0.043)$; specifically, KS resulted in lower systolic blood pressure after the 6-week KS treatment $(F=10.13, p=0.009$, baseline vs. post-intervention $=118.8 \pm 8.9$ mmHg vs. $110.9 \pm 9.7 \mathrm{mmHg}$ ), whereas PLA resulted in no differences $(F=0.04, p=0.851$, baseline vs. postintervention $=111.4 \pm 10.5 \mathrm{mmHg}$ vs. $112 \pm 9.4 \mathrm{mmHg}$ ), as shown in Figure 2. There was no main effect of time for resting diastolic blood pressure $(F=0.07, p=0.788)$, but there was a condition $\times$ time interaction $(F=5.67, p=0.027)$, although a post hoc test did not reveal significance for either condition (KS: $p=0.09$, baseline vs. postintervention $=76.9 \pm 9.3 \mathrm{mmHg}$ vs. $73.5 \pm 10.1 \mathrm{mmHg}$; PLA: $p=0.85$, baseline vs. post-intervention $=68.5 \pm 6.1 \mathrm{mmHg}$ vs. $72.8 \pm 10.6 \mathrm{mmHg}$ ). There was no main effect of time $(F=3.38, p=0.081)$ and no condition $\times$ time interaction ( $F=0.35, p=0.562$; baseline: [KS] $60.9 \pm 15.0$ bpm vs. [PLA] $63.0 \pm 12.5 \mathrm{bpm}$ and post-intervention: [KS] $63.33 \pm 13.16$ bpm vs. [PLA] $67.70 \pm 7.44 \mathrm{bpm}$ ) for resting heart rate.

\section{Comprehensive metabolic panel}

There was a main effect of time for glucose $(F=5.62$, $p=0.028$ ) as glucose was greater post-intervention compared to baseline; however, there was no condition $\times$ time interaction $(F=0.54, p=0.471)$. There were no main effects of time or condition $\times$ time interactions for plasma sodium $(F=0.06, p=0.810$ and $F=0.06, p=0.810$, respectively), potassium $(F=0.05, p=0.826$ and $F=0.45, p=0.512$, respectively), chloride $(F=0.83, p=0.375$ and $F=0.83$,

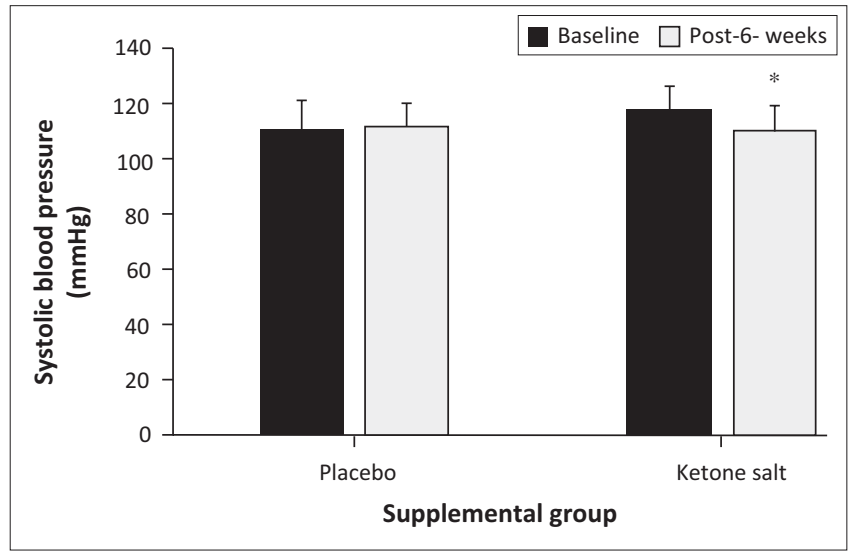

Placebo, $n=10$; ketone salt, $n=13$. Values represent the mean \pm standard deviation $*, p<0.05$ versus baseline

FIGURE 2: Systolic blood pressure before and after taking the placebo or ketone salt supplement daily for 6 weeks. $p=0.375$, respectively $), \mathrm{CO}_{2}(F=1.52, p=0.233$ and $F=1.124$, $p=0.302$, respectively), $\mathrm{BUN}(F=0.00, p=0.955$ and $F=0.73$, $p=0.403$, respectively $)$, creatinine $(F=3.78, p=0.067$ and $F=0.11, p=0.748$, respectively $)$, calcium $(F=2.24, p=0.151$ and $F=0.13, p=0.724$, respectively), total protein $(F=1.28$, $p=0.272$ and $F=0.43, p=0.522$, respectively), albumin $(F=0.06, p=0.802$ and $F=1.03, p=0.323$, respectively), total bilirubin $(F=1.23, p=0.282$ and $F=1.92, p=0.18$, respectively $)$, alkaline phosphatase $(F=1.31, p=0.266$ and $F=2.32$, $p=0.144$, respectively), AST $(F=0.06, p=0.816$ and $F=0.73$, $p=0.404$, respectively) or ALT $(F=0.06, p=0.816$ and $F=0.73, p=0.404$, respectively), as shown in Table 2 .

\section{Urinalysis}

One urine sample in the KS group did not get analysed on time, and therefore the results were invalid and not included. For all other samples, there were no main effects of time or condition $\times$ time interactions for urine levels of glucose (negative levels: $F=1.36, p=0.258$ and $F=1.36, p=0.258$, respectively), ketones (negative levels: $F=2.05, p=0.169$ and $F=0.04, p=0.840$, respectively), blood (negative levels: $F=0.22, p=0.642$ and $F=0.22, p=0.642$, respectively), protein (negative levels: $F=0.04, p=0.840$ and $F=2.05$, $p=0.169$, respectively), leukocytes (negative to trace levels: $F=2.63, p=0.122$ and $F=1.06, p=0.315$, respectively), urobilinogen $(F=1.36, p=0.258$ and $F=1.36, p=0.258$, respectively) or $\mathrm{pH}$ levels $(F=2.89, p=0.106$ and $F=2.89$, $p=0.106$, respectively) (Table 2 ).

\section{Complete blood count}

There was no main effect of time for whole blood $\mathrm{MCH}$ $(F=3.96, p=0.061)$; however, there was a condition $\times$ time interaction $(F=5.11, p=0.036)$; specifically, PLA resulted in lower $\mathrm{MCH}$ after the 6-week PLA treatment $(F=11.58, p=0.009)$, whereas $\mathrm{KS}$ resulted in no differences $(F=0.04, p=0.855)$. There were no main effects of time or condition $\times$ time interactions for WBC $(F=0.51, p=0.484$ and $F=0.20, p=0.659$, respectively), $\operatorname{RBC}(F=0.50$, $p=0.490$ and $F=0.00, p=1.00$, respectively), Hgb $(F=0.00, p=1.00$ and $F=0.45, p=0.51$, respectively $)$, Hct $(F=0.43, p=0.519$ and $F=0.01, p=0.908$, respectively), $\operatorname{MCHC}(F=3.82, p=0.066$ and $F=2.91, p=0.104$, respectively), $\mathrm{MCV}(F=0.11, p=0.748$ and $F=0.78$, $p=0.387$, respectively), $\operatorname{RDW}(F=0.41, p=0.532$ and $F=0.41, p=0.532$, respectively $), \operatorname{PLT}(F=0.09, p=0.763$ and $F=0.00, p=0.966$, respectively $)$ or $\operatorname{MPV}(F=3.73$, $p=0.069$ and $F=0.05, p=0.821$, respectively), as shown in Table 3.

\section{Blood lipid panel}

There were no main effects of time or condition $\times$ time interactions for plasma TC $(F=0.52, p=0.481$ and $F=0.07$, $p=0.790$, respectively), HDLc $(F=0.07, p=0.798$ and $F=1.15$, $p=0.297$, respectively), LDLc $(F=0.62, p=0.440$ and $F=1.12$, $p=0.30$, respectively $)$ or TG $(F=0.07, p=0.802$ and $F=0.49$, $p=0.492$, respectively), as shown in Table 3 . 
TABLE 2: Comprehensive metabolic panel and urinalysis before and after taking the placebo or ketone salt supplement for 6 weeks.

\begin{tabular}{|c|c|c|c|c|}
\hline \multirow[t]{2}{*}{ Measure } & \multicolumn{2}{|c|}{ PLA } & \multicolumn{2}{|c|}{ KS } \\
\hline & Baseline & Post-intervention & Baseline & Post-intervention \\
\hline \multicolumn{5}{|c|}{ Comprehensive metabolic panel (plasma) } \\
\hline Glucose (mg/dL) & $88.0(11.7)$ & $95.4(16.5)$ & $84.8(12.0)$ & $88.7(9.1)$ \\
\hline Sodium (mmol/L) & $138.9(1.5)$ & $138.7(1.1)$ & $138.9(1.5)$ & $138.9(1.4)$ \\
\hline Potassium (mmol/L) & $4.2(0.4)$ & $4.1(0.2)$ & $4.0(0.3)$ & $4.0(0.2)$ \\
\hline Chloride (mmol/L) & $104.8(2.4)$ & $105.6(1.8)$ & $105.1(1.2)$ & $105.1(1.8)$ \\
\hline $\mathrm{CO}_{2}(\mathrm{mmol} / \mathrm{L})$ & $27.7(2.6)$ & $26.6(2.5)$ & $27.4(2.2)$ & $27.3(2.2)$ \\
\hline BUN (mg/dL) & $13.6(4.0)$ & $12.9(4.7)$ & $14.3(5.8)$ & $14.8(5.8)$ \\
\hline Creatinine (mg/dL) & $1.0(0.2)$ & $1.0(0.2)$ & $1.0(0.2)$ & $1.0(0.2)$ \\
\hline Calcium (mg/dL) & $9.4(0.2)$ & $9.3(0.1)$ & $9.5(0.3)$ & $9.5(0.3)$ \\
\hline Total protein (g/dL) & $7.3(0.3)$ & $7.1(0.3)$ & $7.4(0.4)$ & $7.4(0.3)$ \\
\hline Albumin (g/dL) & $4.5(0.2)$ & $4.4(0.3)$ & $4.4(2.2)$ & $4.5(0.2)$ \\
\hline Total bilirubin (mg/dL) & $0.6(0.3)$ & $0.6(0.3)$ & $0.7(0.2)$ & $0.6(0.3)$ \\
\hline Alkaline phosphatase (U/L) & $63.2(12.8)$ & $69.1(9.3)$ & $74.1(13.8)$ & $73.3(16.5)$ \\
\hline $\operatorname{ALT}(U / L)$ & $30.0(11.6)$ & $31.3(12.3)$ & $24.8(11.0)$ & $22.0(7.9)$ \\
\hline \multicolumn{5}{|l|}{ Urinalysis } \\
\hline $\mathrm{pH}$ levels & $6.1(0.9)$ & $6.1(1.0)$ & $6.3(0.7)$ & $7.1(1.1)$ \\
\hline Urobilinogen (EU/dL) & $0.3(0.3)$ & $0.4(0.4)$ & $0.2(0.0)$ & $0.2(0.0)$ \\
\hline
\end{tabular}

Note: Values represent the mean ( \pm standard deviation).

PLA, placebo $(n=10)$; KS, ketone salt $(n=13)$; BUN, blood urea nitrogen; AST, aspartate aminotransferase; ALT, alanine transaminase.

TABLE 3: Complete blood count and lipid panel before and after taking the placebo or ketone salt supplement for 6 weeks.

\begin{tabular}{|c|c|c|c|c|}
\hline \multirow[t]{2}{*}{ Measure } & \multicolumn{2}{|c|}{ PLA } & \multicolumn{2}{|c|}{ KS } \\
\hline & Baseline & $\begin{array}{c}\text { Post- } \\
\text { intervention }\end{array}$ & Baseline & $\begin{array}{c}\text { Post- } \\
\text { intervention }\end{array}$ \\
\hline \multicolumn{5}{|c|}{ Complete blood count (whole blood) } \\
\hline WBC $\left(\times 1000 / \mathrm{mm}^{3}\right)$ & $6.0(1.1)$ & $6.1(0.9)$ & $5.7(1.1)$ & $6.0(1.1)$ \\
\hline $\mathrm{RBC}\left(\times 10^{6} / \mathrm{mm}^{3}\right)$ & $5.0(0.7)$ & $5.1(0.6)$ & $4.6(0.4)$ & $5.1(0.6)$ \\
\hline $\mathrm{Hgb}(\mathrm{g} / \mathrm{dL})$ & $13.9(1.1)$ & $13.8(1.3)$ & $13.5(1.6)$ & $13.6(1.4)$ \\
\hline Hct $(\%)$ & $41.7(3.2)$ & $41.9(3.3)$ & $40.0(3.8)$ & $40.3(3.3)$ \\
\hline $\mathrm{MCHC}(\%)$ & $33.5(0.8)$ & $33.0(0.7)$ & $33.8(1.2)$ & $33.8(1.3)$ \\
\hline $\mathrm{MCH}(p g)$ & $28.2(4.1)$ & $27.7(3.9)^{*}$ & $29.4(2.5)$ & $29.4(2.4)$ \\
\hline $\operatorname{MCV}(f l)$ & $84.2(11.0)$ & $83.9(10.5)$ & $86.8(5.6)$ & $86.9(4.7)$ \\
\hline RDW (\%) & $13.7(1.0)$ & $13.7(1.3)$ & $13.6(1.8)$ & $13.4(1.4)$ \\
\hline $\operatorname{PLT}\left(\times 1000 / \mathrm{mm}^{3}\right)$ & $226.4(68.2)$ & $223.8(55.0)$ & $229.7(49.0)$ & $227.7(41.4)$ \\
\hline MPV (fl) & $9.5(1.0)$ & $9.3(0.9)$ & $8.9(1.0)$ & $8.7(1.0)$ \\
\hline \multicolumn{5}{|l|}{ Lipids (plasma) } \\
\hline $\mathrm{TC}(\mathrm{mg} / \mathrm{dL})$ & $168.0(30.0)$ & $166.2(38.8)$ & $166.9(41.5)$ & $163.0(38.6)$ \\
\hline $\mathrm{HDLc}(\mathrm{mg} / \mathrm{dL})$ & $54.1(14.8)$ & $52.3(10.9)$ & $54.1(10.1)$ & $55.2(10.0)$ \\
\hline LDLc (mg/dL) & $93.6(13.2)$ & $94.7(28.4)$ & $98.7(35.2)$ & $91.1(35.5)$ \\
\hline TG (mg/dL) & $102.1(75.4)$ & $96.1(56.2)$ & $71.1(20.9)$ & $83.9(53.4)$ \\
\hline
\end{tabular}

Note: Values represent the mean ( \pm standard deviation)

PLA, placebo $(n=10)$; KS, ketone salt $(n=13)$; WBC, white blood cells; RBC, red blood cells; $\mathrm{Hgb}$, haemoglobin; Hct, haematocrit; $\mathrm{MCHC}$, mean corpuscular haemoglobin concentration $\mathrm{MCH}$, mean corpuscular haemoglobin; MCV, mean corpuscular volume; RDW, red cell distribution width percent; PLT, platelets; MPV, mean platelet volume; TC, total cholesterol HDLc, high-density lipoprotein cholesterol; LDLc, low-density lipoprotein cholesterol; TG, triglycerides.

\section{Blood ketone levels}

Whole blood $\beta$-HB levels were taken at three time points at both the baseline and post-intervention laboratory visits. The three time points include (1) prior to supplement consumption (pre-supplement), (2) 30-min post-supplement (post-30-min) and (3) 60-min post-supplement (post-60-min). There was a main effect for time $(F=7.90, p=0.000)$ and a condition $\times$ time interaction $(F=23.449, p=0.000)$. Specifically, during the baseline visit for the KS group, post-30-min and post-60min were greater than pre-supplement $(p=0.000$ and $p=0.029$, respectively) and post-30-min was greater than post-60-min $(p=0.036)$ and for the post-intervention visit,

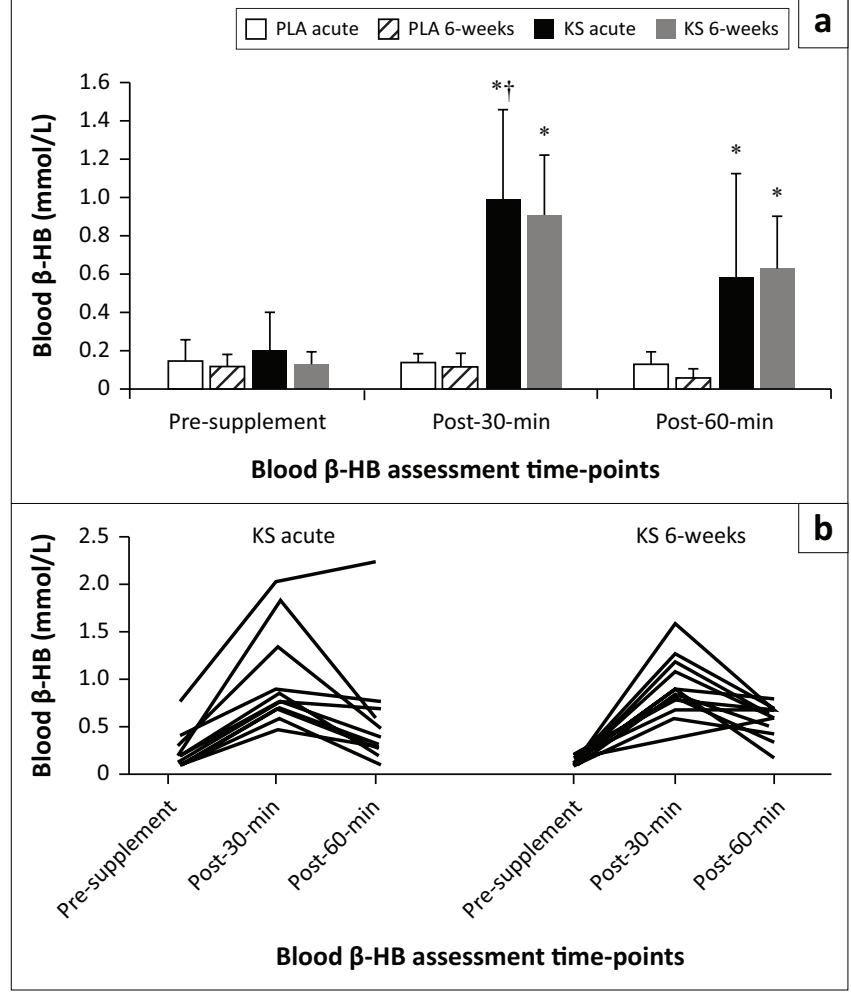

Values for graph (a) represent the mean \pm standard deviation. ${ }^{*}, p<0.05$ versus presupplement; $\dagger, p<0.05$ versus post- $60-\mathrm{min}$. $\mathrm{KS}$, ketone salt.

FIGURE 3: Mean (a) and individual (b) blood ketone levels ( $\beta-\mathrm{HB}$ ) were greater $30 \mathrm{~min}$ (post-30-min) and $60 \mathrm{~min}$ (post-60-min) after consuming ketone salts than immediately before consuming them (pre-supplement). This occurred after consuming only one dose (KS acute) of KS and after regularly consuming KS for 6 weeks (KS 6 weeks).

post-30-min and post-60-min were greater than presupplement ( $p=0.000$ and $p=0.001$, respectively) but post30 -min and post-60-min were not different $(p=0.171)$, as shown in Figure 3. There were no differences $(p>0.05)$ between time points in the baseline or post-intervention laboratory visits for the PLA group. 


\section{Questionnaires}

There were no main effects of time or condition $\times$ time interactions $(p>0.05)$ for any of the descriptors on the POMS or VAS questionnaires.

\section{Discussion}

The findings from this study demonstrate that chronic consumption of $\mathrm{KS}$ is safe for young, healthy adults. Comprehensive metabolic panel, $\mathrm{CBC}$, blood lipids and urine measures remained normal and unaltered after supplementing twice daily with KS for 6 weeks. Furthermore, KS supplementation resulted in reduced systolic blood pressure.

Individuals may opt to use exogenous KS because of difficulties adhering to a KD or because of controversial views revolving around a KD. One such view includes a possible increased risk for kidney complications. For example, Reddy et al..$^{24}$ demonstrated that 6 weeks of a very low-carbohydrate, high-protein diet in healthy males resulted in an increased acid load to the kidney as noted by a reduced urinary $\mathrm{pH}$. However, a very low-carbohydrate, high-protein diet is not a KD per se; a KD typically consists of low-to-moderate protein. With the use of a typical KD consisting of adequate protein and increased hydration, Kossoff et al. ${ }^{25}$ found no association between the diet and kidney complications in children. Nonetheless, our study demonstrates that KS supplementation was shown to be safe for the kidneys; BUN and creatinine, waste products in the blood filtered by the kidneys, were not altered after the 6-week supplementation period which demonstrates sufficient kidney filtration and the acid-base balance was maintained as demonstrated by unaltered blood $\mathrm{CO}_{2}$ and chloride levels, as well as urinary $\mathrm{pH}$ levels.

A second controversial view regarding a $\mathrm{KD}$ includes the possibility for increasing the risk of bone loss because of alterations in electrolyte levels, specifically calcium. ${ }^{26}$ Studies have demonstrated increased urinary calcium excretion as well as actual bone mass loss after incorporation of a very lowcarbohydrate diet primarily in children and adolescents..$^{24,27,28}$ However, again, many of these studies used a dietary ratio consisting of high protein levels..$^{24,28}$ In contrast, the current study suggests that bone mass was maintained with chronic KS supplementation as blood calcium or other electrolyte levels were unaltered; however, bone density measures need to be taken in future studies to ensure no bone loss occurs.

Ketone salt supplementation has also become more common than ketone ester (KE) supplementation for the general public because of the high cost of KE. However, research has demonstrated that $\mathrm{KE}$ supplementation increases blood ketone levels (D- $\beta \mathrm{HB})$ to a greater degree than KS; KE has been shown to increase blood ketone levels to $3 \mathrm{mM}-5 \mathrm{mM}$ after $30 \mathrm{~min}$ of ingestion, whereas the current study demonstrated a 1-mM increase 30 min after KS ingestion. ${ }^{19}$ Also, KE has been shown to be safe and studies have demonstrated that it may improve athletic endurance performance and recovery. ${ }^{14,29,30}$ Further studies are needed to determine whether $\mathrm{KS}$ will benefit athletic performance.

The production of exogenous KS occurs when $\beta-\mathrm{HB}$ is synthesised and bound to sodium as a strategy to improve absorption rate. It is often questioned whether KS supplementation is safe because of the high sodium content. The sodium content for the KS used in this study equalled $914 \mathrm{mg}$ per serving and although that accounts for almost $40 \%$ of the daily recommended sodium content. The participants supplemented twice daily with KS while eating an unrestricted diet. Interestingly, the sodium levels in the blood were unaltered after supplementing with KS for 6 weeks.

The safety of KS supplementation in a healthy, young adult population has been determined herein. In addition to being safe, supplementing with KS positively impacted the cardiovascular system as demonstrated by significant reductions in systolic blood pressure. $\beta$-Hydroxybutyrate has been shown to interact with receptors of the sympathetic nervous system to inhibit sympathetic activity; this may result in reduced blood pressure. ${ }^{31,32}$ For that reason, it would be of interest to examine chronic KS supplementation in a hypertensive population to determine its efficacy as a holistic treatment to reduce blood pressure.

A limitation of this study includes the method of daily supplementation. The participants were supplemented on an honorary system outside of the laboratory, with weekly supplements provided once per week. A specific time schedule with self-reporting measures could help to strengthen participant honesty and reliability outside of the laboratory. In addition, a direct measure to quantify bone mass to provide a more precise determination of bone mass alterations with ketone supplementation would strengthen this study. This study did not specifically control for diet of the participants, but rather whether KS supplementation would impact them on their normal diet. Future research regarding KS supplementation might look at dietary restriction to help challenge or corroborate previous findings that reflect bone loss and kidney function.

\section{Conclusions}

Healthy, young adults were able to maintain normal liver and kidney function, blood lipid levels and other physiological variables pertinent to health after supplementing with KS for 6 weeks, as demonstrated by the CMP, CBC, lipid panel and urinalysis results. Ketone salt supplementation also resulted in systolic blood pressure reductions in a non-hypertensive population.

\section{Transparency declaration}

The lead author affirms that this manuscript is an honest, accurate and transparent account of the study being reported. The reporting of this work is compliant with CONSORT. 
The lead author affirms that no important aspects of the study have been omitted and that any discrepancies from the study as planned have been explained. Trial registration of this study was at www.clinicaltrials.gov (Identifier: NCT03452761).

\section{Acknowledgements}

Prüvit Ventures, Inc. provided input regarding the study design. Neither of the funding sources contributed directly in terms of collection or analysis of data, writing of the manuscript or decision to submit the manuscript for publication. The authors would like to thank the funders for support, AU-CTO and the participants.

\section{Competing interests}

The authors declare that they have no financial or personal relationships that may have inappropriately influenced them in writing this article.

\section{Authors' contributions}

A.M.H. was responsible for the experimental design. A.M.H., A.S.Q., K.N.B. and H.R.B collectively assisted with data collection. A.M.H. analysed the data and drafted the manuscript.

\section{Funding}

Financial support was provided by Prüvit Ventures, Inc. and the College of Education at Augusta University.

\section{Disclaimer}

The information presented in this manuscript is without falsification, manipulation or fabrication. All data are reported accurately and honestly to the best of the authors' knowledge. The views and opinions expressed in this article are those of the authors and not an official position of the funder.

\section{Data availability}

Data sharing is not applicable to this article as no new data were created or analysed in this study.

\section{References}

1. Paoli A, Bianco A, Damiani E, Bosco G. Ketogenic diet in neuromuscular and neurodegenerative diseases. BioMed Res Int. 2014;2014:10p. https://doi. org/10.1155/2014/474296

2. Volek JS, Phinney SD, Forsythe CE, et al. Carbohydrate restriction has a more favorable impact on the metabolic syndrome than a low fat diet. Lipids. 2009;44(4):297-309. https://doi.org/10.1007/s11745-008-3274-2

3. Veech RL. The therapeutic implications of ketone bodies: The effects of ketone bodies in pathological conditions: Ketosis, ketogenic diet, redox states, insulin resistance, and mitochondrial metabolism. Prostaglandins Leukot Essent Fatty Acids. 2004;70(3):309-319. https://doi.org/10.1016/j. plefa.2003.09.007

4. Maalouf M, Rho JM, Mattson MP. The neuroprotective properties of calorie restriction, the ketogenic diet, and ketone bodies. Brain Res Rev. 2009;59(2): 293-315. https://doi.org/10.1016/j.brainresrev.2008.09.002
5. McGarry JD, Foster DW. Regulation of hepatic fatty acid oxidation and ketone body production. Annu Rev Biochem. 1980;49(1):395-420. https://doi.org/ 10.1146/annurev.bi.49.070180.002143

6. Robinson AM, Williamson DH. Physiological roles of ketone bodies as substrates and signals in mammalian tissues. Physiol Rev. 1980;60(1):143-187. https://doi. org/10.1152/physrev.1980.60.1.143

7. Hasselbalch SG, Knudsen GM, Jakobsen JO, Hageman LP, Holm S, Paulson OB. Blood-brain barrier permeability of glucose and ketone bodies during shortterm starvation in humans. Am J Physiol Endocrinol Metab. 1995;268(6):E1161E1166. https://doi.org/10.1152/ajpendo.1995.268.6.E1161

8. Holland AM, Kephart WC, Mumford PW, et al. Effects of a ketogenic diet on adipose tissue, liver, and serum biomarkers in sedentary rats and rats that exercised via resisted voluntary wheel running. Am J Physiol Regul Integr Comp Physiol. 2016;311(2):R337-R351. https://doi.org/10.1152/ajpregu. 00156.2016

9. Yancy WS, Olsen MK, Guyton JR, Bakst RP, Westman EC. A low-carbohydrate, ketogenic diet versus a low-fat diet to treat obesity and hyperlipidemia: A randomized, controlled trial. Ann Intern Med. 2004;140(10):769-777. https:// doi.org/10.7326/0003-4819-140-10-200405180-00006

10. Hyatt HW, Kephart WC, Holland AM, et al. A ketogenic diet in rodents elicits improved mitochondrial adaptations in response to resistance exercise training compared to an isocaloric western diet. Front Physiol. 2016;7:533. https://doi. org/10.3389/fphys.2016.00533

11. Sato K, Kashiwaya $Y$, Keon CA, et al. Insulin, ketone bodies, and mitochondrial energy transduction. FASEB J. 1995;9(8):651-658. https://doi.org/10.1096/ fasebj.9.8.7768357

12. McNally MA, Hartman AL. Ketone bodies in epilepsy. I Neurochem. 2012;121(1):28-35. https://doi.org/10.1111/j.1471-4159.2012.07670.x

13. Paoli A, Rubini A, Volek JS, Grimaldi KA. Beyond weight loss: A review of the therapeutic uses of very-low-carbohydrate (ketogenic) diets. Eur J Clin Nutr. 2013;67(8):789. https://doi.org/10.1038/ejcn.2013.116

14. White $\mathrm{H}$, Venkatesh B. Clinical review: Ketones and brain injury. Crit Care. 2011;15(2):219. https://doi.org/10.1186/cc10020

15. Stubbs BJ, Cox PJ, Evans RD, et al. On the metabolism of exogenous ketones in humans. Front Physiol. 2017;8:848. https://doi.org/10.3389/fphys.2017. 00848

16. KesI SL, Poff AM, Ward NP, et al. Effects of exogenous ketone supplementation on blood ketone, glucose, triglyceride, and lipoprotein levels in Sprague-Dawley rats. Nutr Metab (Lond). 2016;13(1):9. https://doi.org/10.1186/s12986-0160069-y

17. Cox PJ, Clarke K. Acute nutritional ketosis: Implications for exercise performance and metabolism. Extrem Physiol Med. 2014;3(1):17. https://doi.org/10.1186/ 2046-7648-3-17

18. Ari C, Kovács Z, Juhasz G, et al. Exogenous ketone supplements reduce anxiety related behavior in Sprague-Dawley and Wistar Albino Glaxo/Rijswijk rats. Front Mol Neurosci. 2017;9:137. https://doi.org/10.3389/fnmol.2017.00036

19. Clarke K, Tchabanenko K, Pawlosky R, et al. Kinetics, safety and tolerability of (R)-3-hydroxybutyl (R)-3-hydroxybutyrate in healthy adult subjects. Regul Toxicol Pharmacol. 2012;63(3):401-408. https://doi.org/10.1016/j.yrtph.2012. 04.008

20. Plecko B, Stoeckler-Ipsiroglu S, Schober E, et al. Oral $\beta$-hydroxybutyrate supplementation in two patients with hyperinsulinemic hypoglycemia: Monitoring of $\beta$-hydroxybutyrate levels in blood and cerebrospinal fluid, and in the brain by in vivo magnetic resonance spectroscopy. Pediatr Res. 2002; 52(2):301.

21. Valayannopoulos V, Bajolle F, Arnoux JB, et al. Successful treatment of severe cardiomyopathy in glycogen storage disease type III With D, L-3-hydroxybutyrate, ketogenic and high-protein diet. Pediatr Res. 2011;70(6):638. https://doi. org/10.1203/PDR.0b013e318232154f

22. Van Hove JL, Grünewald S, Jaeken J, et al. D, L-3-hydroxybutyrate treatment of multiple acyl-CoA dehydrogenase deficiency (MADD). Lancet. 2003;361(9367): 1433-1435. https://doi.org/10.1016/S0140-6736(03)13105-4

23. Shacham S. A shortened version of the Profile of Mood States. J Pers Assess. 1983;47(3):305-306. https://doi.org/10.1207/s15327752jpa4703_14

24. Reddy ST, Wang CY, Sakhaee K, Brinkley L, Pak CY. Effect of low-carbohydrate high-protein diets on acid-base balance, stone-forming propensity, and calcium metabolism. Am J Kidney Dis. 2002;40(2):265-274. https://doi.org/10.1053/ ajkd.2002.34504

25. Kossoff EH, Pyzik PL, Furth SL, Hladky HD, Freeman JM, Vining EP. Kidney stones, carbonic anhydrase inhibitors, and the ketogenic diet. Epilepsia. 2002;43(10): 1168-1171. https://doi.org/10.1046/j.1528-1157.2002.11302.x

26. Hahn TJ, Halstead LR, DeVivo DC. Disordered mineral metabolism produced by ketogenic diet therapy. Calcif Tissue Int. 1979;28(1):17-22. https://doi.org/ 10.1007/BF02441213

27. Bergqvist AC, Schall JI, Stallings VA, Zemel BS. Progressive bone mineral content loss in children with intractable epilepsy treated with the ketogenic diet. American J Clin Nutr. 2008;88(6):1678-1684. https://doi.org/10.3945/ajcn. 2008.26099

28. Willi SM, Oexmann MJ, Wright NM, Collop NA, Key LL. The effects of a highprotein, low-fat, ketogenic diet on adolescents with morbid obesity: Body composition, blood chemistries, and sleep abnormalities. Pediatrics. 1998;101(1): 61-67. https://doi.org/10.1542/peds.101.1.61 
29. Cox PJ, Kirk T, Ashmore T, et al. Nutritional ketosis alters fuel preference and thereby endurance performance in athletes. Cell Metab. 2016:24(2):256-268. https://doi.org/10.1016/j.cmet.2016.07.010

30. Holdsworth DA, Cox PJ, Kirk T, Stradling H, Impey SG, Clarke K. A ketone ester drink increases postexercise muscle glycogen synthesis in humans. Med Sci Sports Exerc. 2017:49(9):1789. https://doi.org/10.1249/MSS.000000000000 1292
31. Kimura I, Inoue D, Maeda T, et al. Short-chain fatty acids and ketones directly regulate sympathetic nervous system via G protein-coupled receptor 41
(GPR41). Proc Natl Acad Sci USA. 2011;108(19):8030-8035. https://doi.org/ 10.1073/pnas.1016088108

32. Grassi G. Role of the sympathetic nervous system in human hypertension. J Hypertens. 1998;16(12):1979-1987. https://doi.org/10.1097/00004872-199 816121-00019 